\title{
Radar Data Objective Analysis
}

\author{
R. JefFrey Trapp* and Charles A. Doswell III \\ NOAA/National Severe Storms Laboratory, Norman, Oklahoma
}

(Manuscript received 24 August 1998, in final form 22 February 1999)

\begin{abstract}
The spherical geometry of weather radar scans results in a data distribution wherein datapoint separation in one coordinate direction and/or in one part of the analysis domain can differ widely from that in another. Objective analysis of the nonuniform radar data to a uniform Cartesian grid is desirable for many diagnostic purposes. For the benefit of the diagnostic data analyst as well as of users of these analyses, the authors evaluate properties of techniques typically used for such objective analysis. This is done partly through theoretical consideration of the properties of the schemes, but mostly by empirical testing. In terms of preservation of the phase and amplitude of the input data, predictability of the degree of smoothing and filtering, and relative insensitivity to input data unsteadiness or spatial characteristic, the isotropic Gaussian or Barnes-type weight function with constant smoothing parameter appears to be the most desirable of the schemes considered. Modification of this scheme so that the weight function varies spatially, with the datapoint spacing, results in an improved analysis, according to some commonly used measures of error. Interpretation of analyses based on such a modified scheme can be affected, however. For example, analyses of unsteady input fields suffer from a convolution of the temporal evolution of the data with spatial variations of the weight function. As a consequence, unambiguous assessment of physical evolution is precluded.
\end{abstract}

\section{Introduction}

It is a common practice to merge radar reflectivity factor and Doppler velocity information with data collected from different platforms (e.g., a network of rain gauges) or defined in different physical domains with different origins (e.g., multiple Doppler radars). Some, though not all, schemes for assimilation of radar data into numerical models as initial and/or boundary conditions require some level of objective analysis (e.g., Shapiro et al. 1996). With these and numerous other applications of weather radar, it is desirable to map the radar data from their original locations in the spherical coordinate system of the radar to a uniform grid of a common (typically Cartesian) coordinate system. Smoothing and filtering of the data in some systematic way are inherent in this process of objective analysis (hereafter, OA).

Some attributes of radar data pose a particular challenge to certain OA schemes. For example, the spatial distribution of data points varies continuously across

\footnotetext{
* Additional affiliation: Cooperative Institute for Mesoscale Meteorological Studies, University of Oklahoma, Norman, Oklahoma.

Corresponding author address: Dr. R. Jeffrey Trapp, P.O. Box 3000, NCAR/MMM, Boulder, CO 80307.

E-mail: jtrapp@ncar.ucar.edu
}

the radar domain, and therefore there is no single Nyquist wavelength. Moreover, the data point separation in the radial direction can differ by at least an order of magnitude from the separation in the azimuthal direction (or with respect to consecutive planes at constant elevation angle). Also, data domain coordinate axes typically are not aligned with the Cartesian coordinate basis vectors. The foregoing attributes imply that a meteorological phenomenon that is undersampled in part of the domain may be well-sampled elsewhere. Hence, the analyst is faced with the difficulty of producing an analysis that retains to the fullest extent the input wavelengths or spatial scales that are adequately sampled, yet prevents the retention in the analysis of those wavelengths or scales that are underor marginally sampled.

During the Verification of the Origins of Rotation in Tornadoes Experiment (VORTEX; Rasmussen et al. 1994), recently developed mobile Doppler radars, both airborne (Jorgensen et al. 1996; Wakimoto et al. 1996) and ground based (Wurman et al. 1997; Bluestein et al. 1997), were used extensively for studies of tornadic storms and attendant tornadogenesis. Of course, Doppler radars have long been employed for severe local storms research and warning, and the problems with radar data objective analysis (hereafter, RDOA) are not new. However, the need to push these datasets to their resolution limits in order to maximize detail and re- 
solve $^{1}$ presumed important scales of motion has motivated us to reexamine the current "industry standards" of RDOA.

Despite its importance and ubiquity, RDOA has received relatively little formal treatment other than by Heymsfield (1976) and Mohr and Vaughn (1979), for example. With the removal of computer resource limitations faced by analysts during the era when Doppler weather radar was in its relative infancy, it is possible now to explore and test numerous $\mathrm{OA}$ techniques with considerable thoroughness. We do so in this paper by evaluating theoretical properties (section 2) as well as by empirical testing using analytically defined input data (section 3). The analytic fields have been developed to exhibit some verisimilitude with regard to storm-scale phenomena typically observed by Doppler radar. Since analysis of airborne Doppler radar data collected during VORTEX provided the initial impetus for this study, our experiments use sampling similar to that found in quasi-vertical scans of X-band, airborne Doppler radars mounted in the tails of the NOAA P-3 aircrafts (see Jorgensen et al. 1996). Results of our evaluation are summarized in section 4 .

Note that tests of statistical "optimal" interpolation approaches like that taken by Heymsfield (1976) or of variational approaches like that due to Testud and Chong (1983) or Scialom and Lemaître (1990) are foregone here. This allows us to focus on the commonly used (according to the formal literature) RDOA techniques. With these, a priori data weighting is prescribed with the typical goal of producing smoothed and filtered analyses that are suitable for diagnostic purposes. Our RDOA "tutorial," then, is written with the relatively ubiquitous data analysts in mind, and additionally for the users of such analyses, so that the users can make informed interpretations (recognition of interpolation artifices, etc.) of past and present analyses.

\section{Theoretical spectral responses}

In this section, we evaluate theoretical properties of three objective analysis schemes. Specifically, we are interested in (i) the preservation of the phase and amplitude of the input data and (ii) the predictability of the smoothing and filtering properties; desirable schemes are those that yield predictable results and that preserve the characteristics of the input data. Tools developed in this section are used to design the empirical tests discussed in section 3.

\footnotetext{
${ }^{1}$ For reference herein, wavelengths of motion are said to be "resolved" if sampled by at least 6 to 10 independent radar data points, of datapoint spacing equal to the largest dimension in the radar sample volume (Carbone et al. 1985, and citations therein); datapoint spacing in this largest dimension is considered to be the data resolution.
}

\section{a. Barnes and Cressman weight functions}

The basic process of any weighted average objective analysis scheme can be described by

$$
f_{p}^{\prime}=\sum_{q=1}^{N} w_{q} f_{q} / \sum_{q=1}^{N} w_{q}
$$

where $f_{p}^{\prime}$ is the analyzed value at gridpoint $p, f_{q}$ is the datum at datapoint $q$, and $w_{q}$ is the weight associated with the input datum $f_{q}$. Knowledge of the Fourier transform of $w_{q}$ provides insight into property (i) mentioned above. Treatment of the Fourier transform via integrals, as we are about to do, implies that the function being transformed is continuous (or has, at most, a finite number of discontinuities). Although this is not a limitation when considering weight functions, which typically are specified as continuous functions, it is a distinctly unrealistic assumption when applied to the data, which are known only at discrete points. In principle, however, it is possible to incorporate the discreteness of the data via Dirac Comb functions, if a more accurate approach is desired (see Pauley and Wu 1990). We are interested in only the essential elements and so will ignore this complication in order to simplify the mathematical treatment.

We begin by recalling the Fourier transform pair,

$$
\begin{aligned}
& W(k)=\int_{-\infty}^{\infty} w(x) e^{-i k x} d x \quad \text { and } \\
& w(x)=\frac{1}{2 \pi} \int_{-\infty}^{\infty} W(k) e^{i k x} d k,
\end{aligned}
$$

where $W$ is the Fourier transform of weight function $w$, $k$ is the wavenumber, $x$ is some Cartesian distance in one-dimensional (1D) space, and $i=(-1)^{1 / 2}$. For a twodimensional (2D), isotropic function $w(r)$, Eq. (2.2a) becomes the Hankel transform:

$$
W(K)=2 \pi \int_{0}^{\infty} w(r) J_{0}(K r) r d r
$$

where $r$ is some radial distance, $K$ is the radial wavenumber, and $J_{0}$ is the Bessel function of the first kind, order zero. Following Daley (1991, p. 74), a theoretical response function $D(K)$ can be expressed as

$$
D(K)=W(K) / W(0),
$$

which describes the amplitude response (as a function of wavenumber) produced by the weight function as the data are interpolated.

Using the notation of Koch et al. (1983), the exponential or Barnes (1964) distance-dependent weight function in 2D can be expressed as

$$
w_{q}=\exp \left(-r^{\prime 2} / \kappa\right)
$$

where $r^{\prime}$ is the radial distance separating an analysis point from a data point and $\kappa$ is the smoothing parameter. The Fourier (and Hankel) transform of the Barnes 


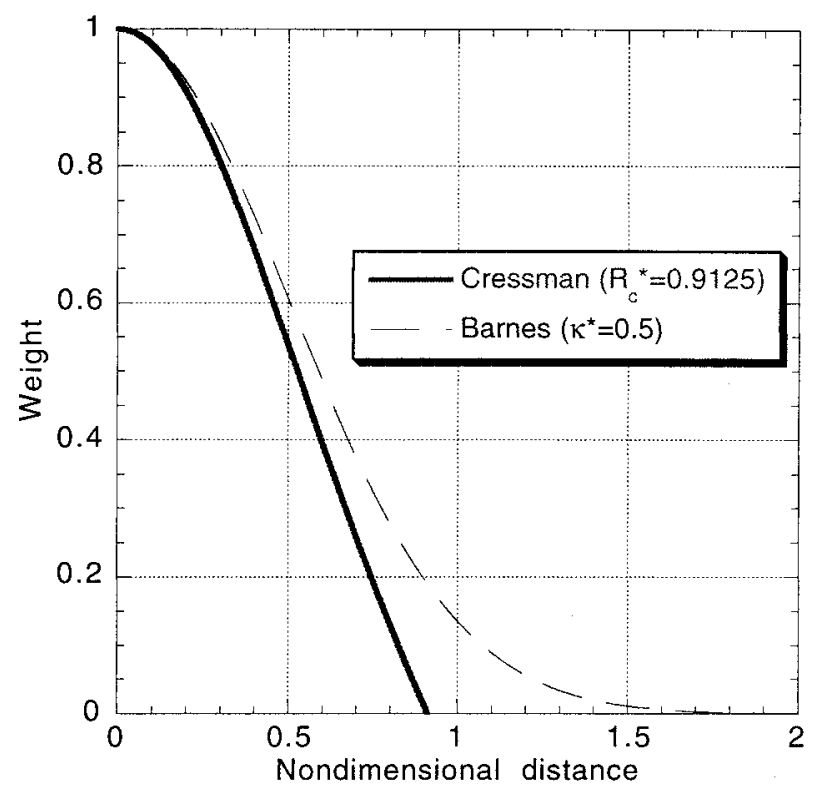

FIG. 1. Evaluation of Barnes weight function with nondimensional smoothing parameter $\kappa^{*}=0.5$ (dashed) and of Cressman weight function with nondimensional influence radius $R_{c}^{*}=0.9125$ (solid).

weight function can be obtained analytically, a convenient property of Gaussian functions. Upon substituting Eq. (2.5) into Eq. (2.3) and then integrating, Eq. (2.4) reveals that,

$$
D(K)=\exp \left[-4 \kappa K^{2} / \pi\right],
$$

or, alternatively,

$$
D\left(\lambda^{*}\right)=\exp \left[-\kappa^{*}\left(\pi / \lambda^{*}\right)^{2}\right],
$$

where $\kappa^{*}=\kappa / L^{2}$ is the nondimensional smoothing parameter, $K=2 \pi / \lambda$, and $\lambda^{*}=\lambda / L$ is the nondimensional wavelength; $L$ is some length scale, which can be chosen to be twice the mean data spacing $(L=2 \Delta$; Koch et al. 1983).

Figure 1 shows the Barnes weight function with $\kappa^{*}$ $=0.5 ;$ the associated theoretical response is illustrated in Fig. 2. Clearly, input waves of nondimensional length equal to unity (or dimensional length $2 \Delta$, the Nyquist wavelength) essentially will be filtered from the analysis. Original amplitudes of longer waves will be damped but still included in the analysis. For example, $75 \%$ of the original amplitudes of waves with length $8 \Delta$ will be retained (Fig. 2).

A more commonly used $w$ in RDOA (as gauged by frequency of references in the meteorological literature) is that due to Cressman (1959). The Cressman weight function can be expressed as

$$
w_{q}= \begin{cases}\frac{R_{c}^{2}-r^{\prime 2}}{R_{c}^{2}+r^{\prime 2}}, & r^{\prime} \leq R_{c} \\ 0, & r^{\prime}>R_{c},\end{cases}
$$

where $R_{c}$ is the radius of influence. Since it is not pos-

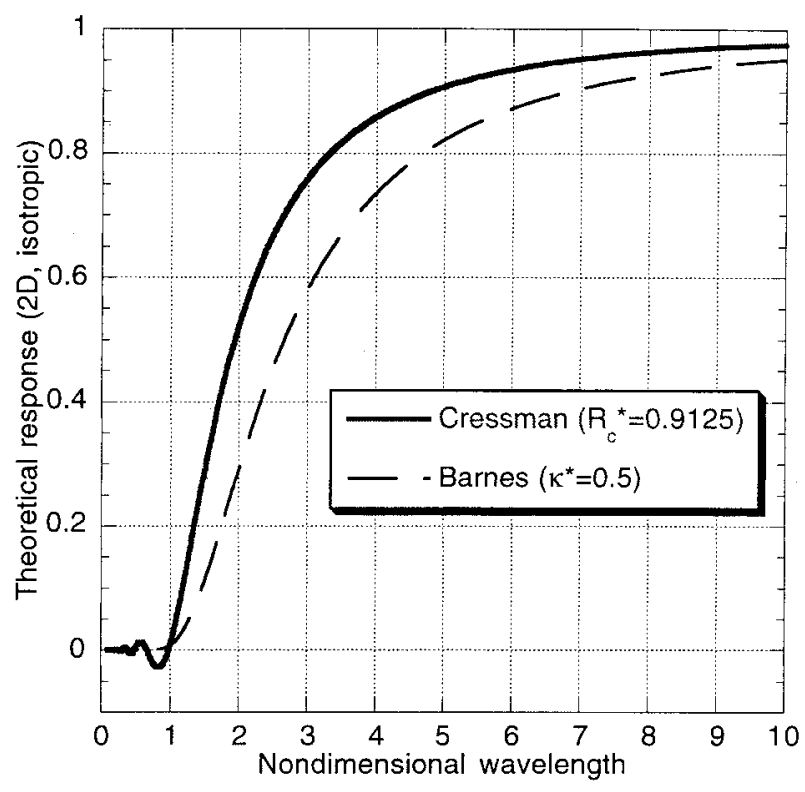

FIG. 2. Theoretical spectral response of Barnes weight function with nondimensional smoothing parameter $\kappa^{*}=0.5$ (dashed) and of Cressman weight function with nondimensional influence radius $R_{c}^{*}$ $=0.9125$ (solid).

sible to find the Fourier transform of the Cressman weight function analytically, the transform must be obtained by numerical integration. We do so herein using the extended Simpson's rule, with a polynomial approximation of $J_{0}$ (Press et al. 1986, p. 223) for the computation of the Hankel transform.

The resulting theoretical response for the Cressman weight function with a nondimensional influence radius of $R_{c}^{*}=0.913$, where $R_{c}^{*}=R_{c} / L$, is also nearly zero at $2 \Delta$ wavelengths (Fig. 2). The Cressman response curve is comparatively steeper. This allows a greater percentage of the amplitudes of all wavelengths, especially the shorter wavelengths, to be retained. At wavelengths less than $2 \Delta$, however, the response oscillates about zero, resulting in negative "sidelobes." Such oscillations are comparable to the so-called Gibbs phenomenon associated with nonperiodic boundary values and owe their existence to the first-order discontinuity (i.e., a discontinuity in the first derivative) of the Cressman weight function at $r=R_{c}$ (see Fig. 1).

We pause here to examine the effects of a negative response. The RDOA process resulting in the analyzed field $f^{\prime}(x)$ can be thought of as a convolution of the input field $f(x)$ with the weight function $w(x)$ :

$$
f^{\prime}(x)=f(x) * w(x) .
$$

By the convolution theorem, the Fourier transform of the convolution, $F^{\prime}(k)$, is equal to the product of the respective Fourier transforms of $f(x)$ and $w(x)$, that is,

$$
F^{\prime}(k)=F(k) W(k) .
$$

Assume that the input data $f(x)$ have a Fourier transform denoted by $F(k)$, which is in general a complex quantity: 


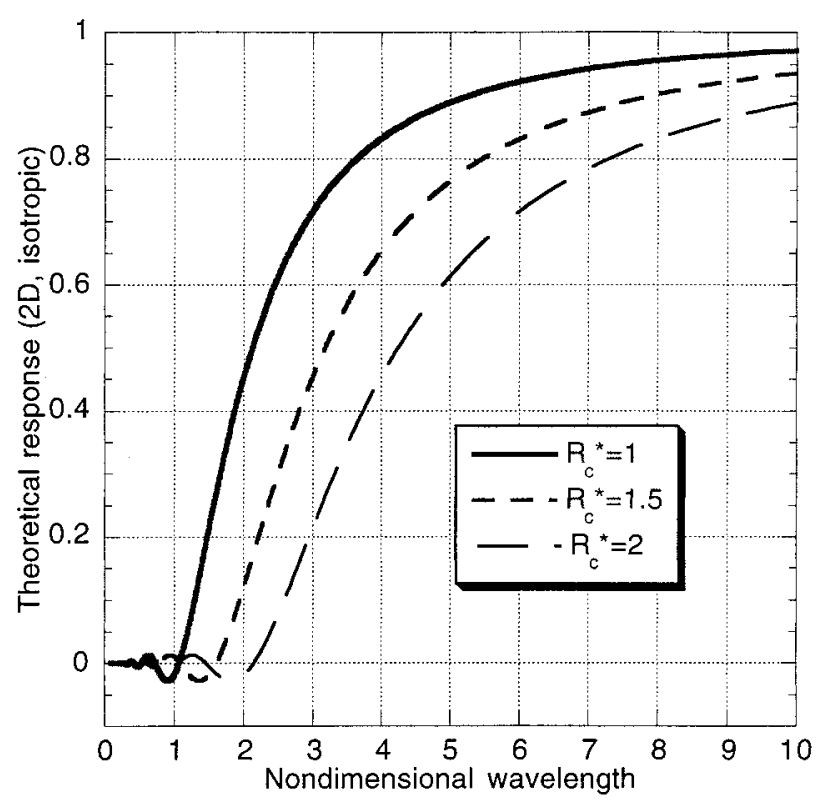

FIG. 3. As in Fig. 2, except with the Cressman weight function with influence radii $R_{c}^{*}=1.0,1.5$, and 2.0 .

$$
F(k)=F_{\mathrm{RE}}(k)+i F_{\mathrm{IM}}(k)=|F(k)| e^{i \alpha(k)},
$$

where subscripts RE and IM denote real and imaginary parts, respectively, $\alpha(k)$ is the phase angle, and $|F(k)|$ is the nonnegative amplitude of the spectrum of the input data. The Fourier transform the weight function also is complex:

$$
W(k)=W_{\mathrm{RE}}(k)+i W_{\mathrm{IM}}(k)=|W(k)| e^{i \psi(k)},
$$

where $\psi(k)$ and $|W(k)|$ are the phase and nonnegative amplitude, respectively, due to the weight function. If response $D(k)$ is negative, as in the example in Fig. 2, $W(k)$ also must be negative, implying in turn that the phase angle $\psi(k)$ must be nonzero. In this case, we have, using Eqs. (2.11) and (2.12) in (2.10),

$$
F^{\prime}(k)=|F||W| e^{i(\alpha+\psi)},
$$

or in words, an analysis whose phase in spectral space is the sum of that due to the input field and the weight function. The weight function ideally should affect only the amplitude of the input field, so certainly such a phase-shifted analysis is undesirable.

Figure 3 reveals that the negative sidelobes and consequential phase shifts occur at progressively larger wavelengths as the Cressman influence radius increases. A heavily smoothed analysis, therefore, is more apt to suffer from phase shifts of resolvable wavelengths $(L / 2 \Delta$ $>1$ ), whereas a lightly smoothed analysis is more susceptible to up-scale aliasing of unresolved wavelengths $(L / 2 \Delta<1)$. This sort of dilemma is not uncommon in OA, where competing effects make the choice of the analysis parameter(s) challenging. Empirical tests presented in the next section disclose some curious properties of the Cressman response's sidelobes.

\section{b. Application to data of variable spacing}

The analytic form of the spectral response of the Barnes weight function makes the Barnes scheme amenable to an exercise that explores the effects of nonuniform data spacing. We apply the Barnes weight function to a hypothetical one-dimensional (or two-dimensional isotropic) data distribution with a monotonically increasing datapoint spacing. The distribution is likened to the change in azimuthal spacing that increases linearly with radar range.

Recall that Eq. (2.7) provides knowledge of the filtering properties of $w$, as used in the Barnes scheme, for a specific nondimensional smoothing parameter $\kappa^{*}$ and nondimensional wavelength $\left(\lambda^{*}\right)$. This allows the analyst to choose, irrespective of the datapoint spacing, those nondimensional wavelengths that will be removed from or retained in the analysis. ${ }^{2}$

In the application of a (single pass) Barnes objective analysis scheme suggested by Koch et al. (1983), $\kappa^{*}$ is chosen based on a desired theoretical response (as in Fig. 2), dimensionalized with some measure of the $a v$ erage $\Delta$, and then used to define the Barnes weight function [Eq. (2.5)]. Subsequently, the data are interpolated irrespective of any variations in $\Delta$ that occur throughout the domain. Such an approach becomes complicated when radar data are involved: "average datapoint spacing" is not meaningful since the azimuthal spacing $\left(\Delta_{\mathrm{az}}\right)$ has a systematic increase with range. In such cases, we recommend that $\kappa^{*}$ be dimensionalized by the maximum spacing of data affecting the analysis domain. In doing so, we are expressing a "conservative" viewpoint: some signal may be lost through smoothing in order to avoid undersmoothing the data in regions of the most coarse datapoint spacing.

To explore the implications of this recommendation, consider a smoothing parameter $\kappa^{*}=0.5$ dimensionalized by the maximum $\Delta_{\mathrm{az}}$. The azimuthal data spacing varies with range according to

$$
\Delta_{\mathrm{az}} \approx R \Delta \theta,
$$

where $R$ is radar range, and $\Delta \theta$ is the (angular) azimuthal data sampling interval. ${ }^{3}$ The azimuthal sampling interval is a function of the antenna rotation rate, pulse repetition frequency, and number of signal pulses, and is independent of the half-power beamwidth (e.g., Brown and Wood 1998). The nominal value of $\Delta \theta$ for the Weather Surveillance Radar-1988 Doppler (WSR-88D) is $1^{\circ}$, and for recent airborne Doppler radar applications,

\footnotetext{
${ }^{2}$ Note that this is valid strictly for isotropic weighting of a continuous data distribution, as mentioned earlier. Equation (2.7) will only approximate the spectral response when applied to discrete, and perhaps nonuniformly, spaced data. For a discussion of the spectral response of a weighted discrete, uniform, data distribution, the reader is referred to Pauley and Wu (1990).

${ }^{3}$ For convenience, values of $\Delta \theta$ are given in degrees. When used in Eq. (2.14), however, these values are converted to radians.
} 


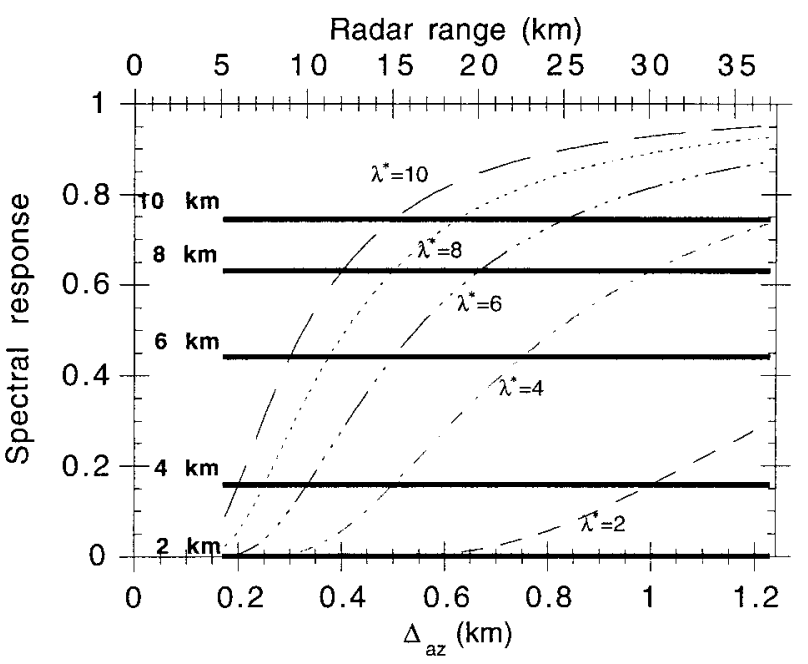

FIG. 4. Theoretical response for Barnes weight function with constant smoothing parameter $\kappa=2.987 \mathrm{~km}^{2}$, as a function of the local data spacing $\Delta_{\mathrm{az}}$. Responses $D$ for dimensional scales are indicated by the solid curves and are constant with respect to changes in $\Delta_{\text {az }}$ (which is a function of the radar range). Responses $D$ for locally nondimensionalized scales (dashed curves) are recomputed for each $\Delta_{\mathrm{az}}$ value by using in Eq. (2.7) $\kappa^{*}=\kappa /\left[\left(2 \Delta_{\mathrm{az}}\right)^{2}\right]$, where $\kappa=2.987$ $\mathrm{km}^{2}$.

$1^{\circ}<\Delta \theta<2^{\circ}$ (e.g., Jorgensen et al. 1996; Hildebrand et al. 1996). To exaggerate somewhat the range-varying azimuthal datapoint spacing, we assign $\Delta \theta=2^{\circ}$, and assume that the maximum range of data points affecting the hypothetical analysis is $R=35 \mathrm{~km}$ (as typical during VORTEX). Using Eq. (2.14), we have $\max \left(\Delta_{\mathrm{az}}=1.22\right.$ $\mathrm{km}$, from which we compute $\kappa=\kappa^{*}\left[\left(2 \Delta_{\mathrm{az}}\right)_{\mathrm{max}}^{2}\right]=2.987$ $\mathrm{km}^{2}$.

Because $\kappa$ is constant, the spectral response of a given dimensional scale or wavelength is independent of radar range and hence datapoint spacing (Fig. 4). It follows that the RDOA scheme damps or removes dimensional scales regardless of how they are resolved. Using $\kappa=$ $2.987 \mathrm{~km}^{2}$, we can recompute a "local" $D$ with $\kappa^{*}=$ $\kappa /\left[\left(2 \Delta_{\mathrm{az}}\right)^{2}\right]$, the local data spacing $\Delta_{\mathrm{az}}$ (which is a function of the radar range), and Eq. (2.7). This results in theoretical responses for locally nondimensionalized wavelengths $\lambda^{*}$ that change with respect to changes in $\Delta_{\text {az }}$ (Fig. 4).

The extent to which physical scales are sampled by the local data resolution is also illustrated in Fig. 4. For example, at a range of $5 \mathrm{~km}$ (where the local data resolution is $0.2 \mathrm{~km}$ ), a 4-km wavelength is sampled by 21 data points (i.e., represented as a " $20 \Delta$ " wave, with $\lambda^{*}=10$ ). At a range of $\sim 30 \mathrm{~km}$ (where the local data resolution is $1.0 \mathrm{~km}$ ), a $4-\mathrm{km}$ wavelength is sampled by five data points (i.e., represented as a " $4 \Delta$ " wave, with $\lambda^{*}=2$ ), hence, it is only marginally resolved. At all ranges, only $19 \%$ of the amplitude of input waves of 4-km length are admitted into the analysis. This exercise exemplifies the "traditional" application of a distantdependent weighted-averaging (DDWA) scheme in

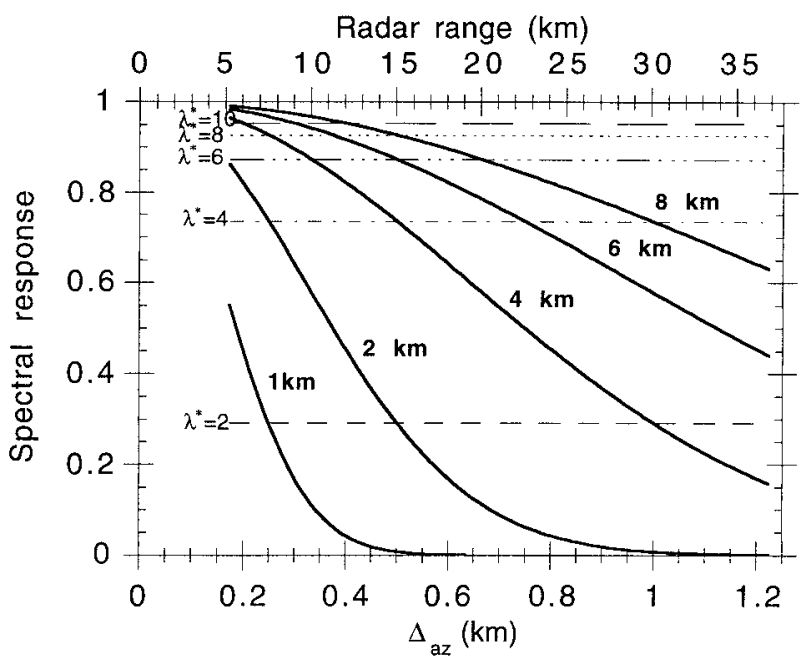

FIG. 5. Theoretical response for Barnes weight function with nondimensional smoothing parameter $\kappa^{*}=0.5$, as a function of the local data spacing $\Delta_{\text {az }}$. Responses $D$ for dimensional scales (solid curves) are recomputed locally using $\kappa^{*}=0.5, \Delta_{\mathrm{az}}$, and $\kappa=\kappa^{*}\left[\left(2 \Delta_{\mathrm{az}}\right)^{2}\right]$ in Eq. (2.7), and changes with respect to changes in $\Delta_{\text {az }}$. Responses $D$ for locally nondimensionalized scales (dashed curves) are constant with respect to changes in $\Delta_{\mathrm{az}}$.

which the free parameter in the weight function (e.g., Cressman influence radius or the Barnes smoothing parameter) is constant.

\section{c. A modified Barnes weight function}

It has been suggested that the free parameter and thus the weight function be allowed to adjust in some way to the local data spacing [e.g., Nelson 1980 (Cressman); Askelson 1996 (Barnes)]. The obvious advantage of this approach is that physical scales are filtered and damped according to how well they are sampled locally. In radar data, this allows a certain physical scale to be damped only slightly when it is well resolved near the radar, yet filtered heavily when it is poorly resolved at a large distance from the radar.

Continuing our simple exercise, consider a "modified" Barnes OA weight function, $w_{q}=w_{q}\left(r^{\prime}, \kappa^{*}, \Delta\right)$, in which the smoothing parameter is redefined everywhere in the analysis domain according to the local data spacing:

$$
\kappa=\kappa^{*}(2 \Delta)^{2},
$$

where $\kappa^{*}$ is fixed and chosen a priori. In this case, nondimensional wavelengths have spectral responses that do not change with data resolution (Fig. 5). A 2-km wavelength is treated as a " $4 \Delta^{\prime} "\left(\lambda^{*}=2\right)$ wave at a range of $15 \mathrm{~km}(0.52-\mathrm{km}$ data spacing), and consequently damped heavily. However, because it is sampled as a " $12 \Delta^{\prime} "\left(\lambda^{*}=6\right)$ wave at a range of $5 \mathrm{~km}(0.17$ $\mathrm{km}$ data spacing), a $2-\mathrm{km}$ wavelength observed at this range retains most of its amplitude in the analysis. As 
is shown in the following section, this approach may not be well suited for all applications.

\section{Empirical testing}

We now employ an empirical methodology to evaluate the results from different OA schemes. Continuous, error-free, analytic fields are sampled at discrete data points defined in polar coordinates $(R, \theta)$, simulating constant-elevation-angle radar scans of some meteorological scalar. The analytic fields have different input wavelengths and are steady state in all but two sets of experiments. Discretely sampled "data" are mapped via OA onto a uniform Cartesian grid, and the subsequent analysis is compared to the analytic field. Empirical tests involve DDWA schemes with a Barnes weight function (modified and "traditional") and a Cressman weight function, ${ }^{4}$ bilinear interpolation (also a DDWA scheme), and assignment of a gridpoint value using the spatially closest datapoint value (henceforth, "nearest neighbor" analysis). Most of these have been employed by radar data diagnosticians [recently, e.g., by Trapp 1999 (Barnes); Wakimoto and Liu 1998 (Cressman); Mohr and Vaughn 1979 (bilinear interpolation)].

With admitted loss of generality to multidimensional OA except in limited circumstances, our tests are confined to two dimensions. This approach allows us to perform a suite of idealizations economically, and interrogate them without the complications of a third dimension. The realm of issues related to $\mathrm{OA}$ with dimensionality higher than two is beyond the scope of this paper. Nevertheless, results presented here reveal basic characteristics of schemes that should be known prior to a scheme's application to more than two dimensions.

\section{a. Steady-state analytic fields}

As in the one-dimensional exercise above, azimuthal data spacing varies with range according to $\Delta_{\mathrm{az}} \approx R \Delta \theta$. Our hypothetical radar has an azimuthal sampling interval of $\Delta \theta=2^{\circ}$ and a range-gate spacing $\Delta R=0.075$ $\mathrm{km}$. We assume that the radar "scans" at an elevation angle of $0^{\circ}$ (or, alternatively, consider a single, quasivertical scan or "sweep" of an airborne Doppler radar). These radar attributes dictate the discretization of the continuous input field:

$$
f(x, y)=A \cos \left(\frac{2 \pi}{L_{x}} k x\right) \sin \left(\frac{2 \pi}{L_{y}} l y\right)
$$

\footnotetext{
${ }^{4}$ A "modified Cressman" scheme, analogous in design to the modified Barnes scheme discussed in section $2 \mathrm{c}$, was developed and tested as well. The results of empirical tests applied to the modified Cressman scheme paralleled those of the modified Barnes scheme in terms of rms error (though slightly less in the former) and inherent shortcomings (susceptibility to interpretation problems, etc.). For economy of presentation, a discussion of such results is omitted here.
}

where $x=R \sin \theta-x_{R} ; y=R \cos \theta-y_{R} ; A$ is the constant amplitude set equal to $10 ; L_{x}=L_{y}=30 \mathrm{~km}$ are the fundamental wavelengths in the $x$ and $y$ directions, respectively; and $k(l)$ is a domain wavenumber index in the $x(y)$ direction. This analytic field represents a checkerboard pattern of circular maxima and minima. Note that the radar is arbitrarily positioned ${ }^{5}$ at $x_{R}, y_{R}=$ $15,-5 \mathrm{~km}$ with respect to a $0 \leq x, y \leq 30 \mathrm{~km}$ Cartesian analysis domain of $\delta_{x}=\delta_{y}=0.5$-km gridpoint spacing. Given this particular domain size and radar position, the maximum datapoint spacing (based on the maximum range affecting the analysis $)$ is $\max \left(\Delta_{\mathrm{az}}, \Delta_{R}\right)=\Delta_{\max }=$ $1.33 \mathrm{~km}$.

In the first set of tests, indices $k$ and $l$ are varied over a wide range of values to illustrate how each scheme responds to different input wavelengths. The resultant data fields are interpolated to the Cartesian grid using $\kappa^{*}=0.5$ in the traditional and modified Barnes weight functions and $R_{c}^{*}=0.913$ in the Cressman weight function [note that both of these parameters yield a Nyquist response $\left.D\left(\lambda^{*}=1\right)=0.007\right]^{6}$; unless otherwise indicated, weighting is applied isotropically. We adhere to our earlier recommendation that for traditional Barnes (Cressman) OA applications, $\kappa^{*}\left(R_{c}^{*}\right)$ should be dimensionalized by the maximum datapoint spacing $\Delta_{\max }=$ $1.33 \mathrm{~km}$, thereby yielding in this case $\kappa=3.538 \mathrm{~km}^{2}$ $\left(R_{c}=2.427 \mathrm{~km}\right)$. Again, the smoothing parameter in the modified Barnes weight function is defined anew for each datum via $\kappa=\kappa^{*}(2 \Delta)^{2}$, where $\Delta$ is evaluated locally as $\Delta=\max \left(\Delta_{\mathrm{az}}, \Delta R\right)$. For comparison, the nearest-neighbor and bilinear interpolation methods also are employed.

For each wavenumber examined, root-mean-square (rms) differences between the objectively analyzed fields and discretized analytic function are lower in the modified Barnes analyses than in the traditional Barnes and Cressman analyses (Fig. 6). Mean absolute errors (MAEs, not shown) are consistent with this result. Objective analysis by the nearest-neighbor and bilinear interpolation methods yields rms differences that are lower still. This measure of error is misleading, however, since the nearest-neighbor and bilinear interpolation analyses are clearly inferior. As depicted by difference fields,

$$
d(x, y)=f(x, y)_{\text {discrete }}-f(x, y)_{\text {analyzed }},
$$

\footnotetext{
${ }^{5}$ The radar origin is located outside the analysis domain to simulate a typical analysis situation using airborne Doppler radar data collected during VORTEX.

${ }^{6}$ We note that the Barnes smoothing parameter $\kappa^{*}=0.5$ and Cressman radius of influence $R_{c}^{*}=0.913$ are chosen because of their very low theoretical response at $2 \Delta$. It is possible with these idealized tests, however, to find (typically smaller) $\kappa^{*}$ and $R_{c}^{*}$ that result in less analysis error for a given input field. Use of smaller $\kappa^{*}$ and $R_{c}^{*}$ in real data $\mathrm{OA}$ applications allow for the possibility that marginally or underresolved wavelengths will adversely affect the analysis.
} 


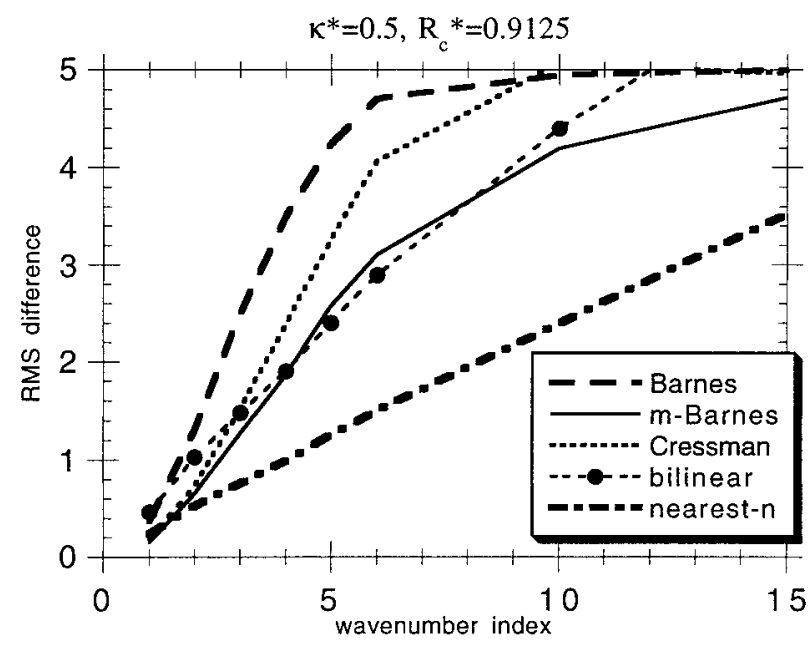

FIG. 6. Root-mean-square (rms) differences between the analytic function and objectively analyzed fields produced by means of the Barnes, modified Barnes, Cressman, bilinear, and nearest-neighbor OA schemes. The abscissa is the wavenumber index $k(=1)$ used in Eq. (3.1) to define the input field.

the nearest-neighbor and bilinear schemes generate "noise," or smaller-scale waves not present in the input data (see Figs. 7e-f); this is a clearly undesirable characteristic of these two schemes. The difference fields also show the range dependency inherent in the modified Barnes scheme: amplitude errors [and hence $d(x, y)$ ] increase with increasing range and datapoint spacing. As illustrated more dramatically in the next section, the potential is high for misinterpreting the range-dependent analysis of the range-independent input field. Note that part of the analysis error depicted in Figs. 6 and 7 can be explained by the range-dependent sampling and discretization of the input field, rather than by shortcomings of a particular OA scheme. In other words, the analytic function is discretized more coarsely (gradients and amplitudes less resolved) as range, and thus azimuthal spacing, increases.

Discrete Fourier transforms are used to examine the analyses as represented in vector wavenumber $(k, l)$ space. Two-dimensional spectra $F(k, l)$ are determined following Errico (1985). ${ }^{7}$ For convenience of presentation, we compute $1 \mathrm{D}$ spectra $I(K)$ by summing the 2D spectra within discrete annuli with central radii $K=\left(k^{2}+l^{2}\right)^{1 / 2}$ [see Errico 1985, Eq. (5)]. The 1D spectra then are normalized by the maximum of $I(K)$ calculated from $f(x, y)_{\text {analyzed }}$, to approximate a response function.

Consider input data with $k=l=2$ and $k=l=4$, corresponding to $K=3$ and $K=6$, respectively. A peak in the normalized $1 \mathrm{D}$ spectra at the input wavenumber

\footnotetext{
${ }^{7}$ Since our application involves periodic functions (analyzed onto a regularly spaced grid), removal of trends as in Errico (1985) is not required.
}

is indicated for analyses produced by each OA scheme (Fig. 8). Consistent with the theoretical response curves in Fig. 2, input waves of smaller length are damped more heavily, particularly with the Barnes weight with $\kappa=3.538 \mathrm{~km}^{2}$. In terms of maintaining the spectral fidelity of the input data, the nearest-neighbor and bilinear schemes are markedly inferior as mentioned above. Indeed, a spectral broadening is associated with these schemes, and, curiously, with the modified Barnes scheme as well; we have no ready explanation for the spectral broadening associated with the latter.

We next test the sensitivity of the OA techniques to less-idealized, asymmetric input data. These data are defined using Eq. (3.1) for a range of wavenumber index aspect ratios $(=k / l)$; a checkerboard pattern of elliptical maxima and minima results (Fig. 9). Figure 10 depicts the rms differences between the asymmetric, analytic functions and corresponding objectively analyzed fields. A technique that is insensitive to asymmetries (like one with fixed, isotropic Barnes or Cressman weighting) yields rms differences that are symmetric about $k / l=$ 1 and are the same regardless if the input ellipses have major axes parallel to the $x$ axis or parallel to the $y$ axis. The remaining schemes, which exploit the greater resolution in the radial direction, are more successful (have less error) in the cases $k / l<1$ wherein sharp gradients are directed parallel to many radials. In cases where $k / l$ $>1$, these same schemes produce poor analyses because of their inability to maintain the sharp gradients directed normal to many radials (e.g., see Fig. 9).

As shown in the previous section, the theoretical response due to the Cressman weight function has negative sidelobes which, according to theory based on continuous data distributions, should result in a shift of the phase of the input data [see Eq. (2.13)]. We now explore this theoretical effect using additional experiments with input data described by Eq. (3.1).

Analyses are generated using the Cressman weight function with nondimensional influence radii $R_{c}^{*}=$ $0.913,1.0,1.5$, and 2.0. Once dimensionalized by $2 \Delta_{\max }$ $=2.66 \mathrm{~km}$, these influence radii become $R_{c}=2.43$, $2.66,3.99$, and $5.32 \mathrm{~km}$. Note that each influence radius is associated with a theoretical response that first becomes zero at some wavelength $\lambda_{0} \geq 2 \Delta$, yet may oscillate about zero at wavelengths $\lambda<\lambda_{0}$ (see Figs. 2 and 3). An input field with $k=l=12$ in Eq. (3.1) is used; this yields input waves of length $2.5 \mathrm{~km}$, which approximates $2 \Delta_{\max }$.

Figure 11 summarizes experiments with three Cressman radii of influence and discloses an unexpected property of the Cressman weight function: wavelengths that are removed when a relatively narrow weight function (with $R_{c}=2.43 \mathrm{~km}$ ) is used, are "readmitted" into the analysis when a slightly broader weight function (with $R_{c}=2.66 \mathrm{~km}$ ) is applied. This presumably is a manifestation of the response sidelobes and appears not to be a function of their sign. The amplitudes of the readmitted waves are small in these examples, $\sim 2$ orders 

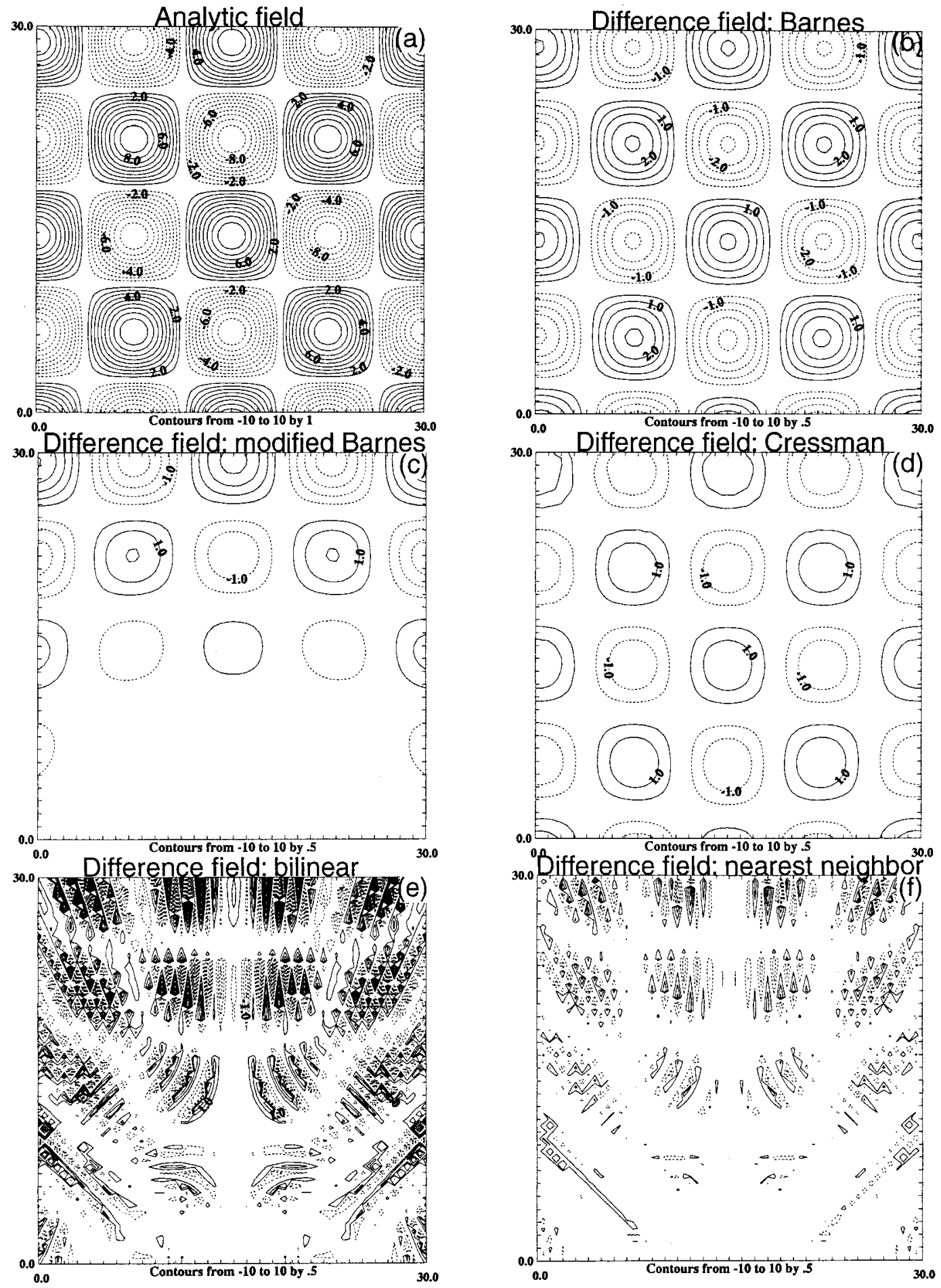

FIG. 7. (a) Discrete evaluation of Eq. (3.1) for the case $k=l=2$. Difference fields, $d(x, y)=f(x, y)_{\text {discrete }}-f(x, y)_{\text {analyzed }}$, between the discretized, exact function and analyses produced by the (b) Barnes, (c) modified Barnes, (d) Cressman, (e) bilinear, and (f) nearest-neighbor OA techniques. In this and all other contour plots, contour interval is given below each plot, solid (dashed) contours indicate positive (negative) values, and the zero contour is omitted. 

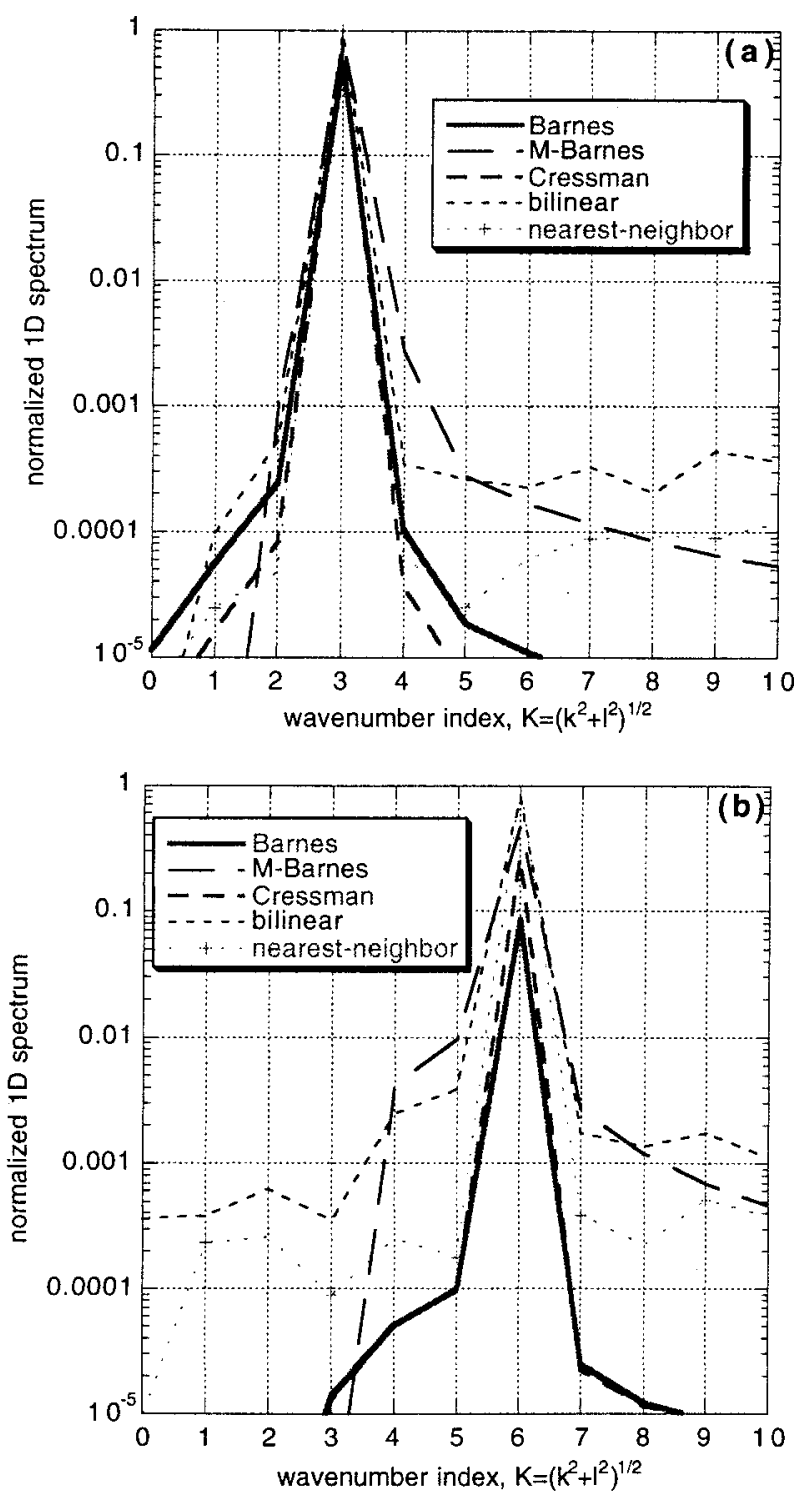

FIG. 8. Normalized 1D spectra as a function of radial wavenumber $K$, for each analysis, given input data defined by (a) $k=l=2$ and (b) $k=l=4$ in Eq. (3.1).

of magnitude less than the maximum amplitude of the input field. Note that the analyzed field in the domain corners can be attributed to aliasing.

Difference fields (not shown) fail to reveal any discernible phase differences between the input field and analyses. In light of the work of Pauley and Wu (1990), it is plausible that the actual response is sufficiently different from the continuous, theoretical response; thus, the negative sidelobes and theorized phase shift (cf. section 2a) occur at a wavelength(s) not represented in the input field used in the Fig. 11 experiments. Full exploration of this artifact of the Cressman weight function is beyond the scope of this paper, but may be the subject of forthcoming research.

\section{b. Unsteady analytic fields}

The following series of experiments is used to examine the behavior of certain OA schemes when applied to an unsteady problem. We treat the unsteady field as an isolated phenomenon (e.g., a thunderstorm) that moves through the domain of nonuniform datapoint spacing. Since radar data analysts often are faced with situations of storms or storm complexes moving through the domain, these experiments are particularly relevant and show more dramatically the potential pitfalls of using RDOA schemes with spatially varying weight functions.

Consider the positive function (henceforth, "storm"):

$$
g(x, y, t)= \begin{cases}B \cos \left(2 \pi r^{\prime}\right), & r^{\prime}<1 / 4 \\ 0, & r^{\prime} \geq 1 / 4\end{cases}
$$

where

$r^{\prime}=\left\{\left(\frac{m}{L}\right)^{2}\left[x-\left(\hat{x}+C_{x} t\right)\right]^{2}+\left(\frac{n}{L}\right)^{2}\left[y-\left(\hat{y}+C_{y} t\right)\right]^{2}\right\}^{1 / 2}$,

$B=10$ is the constant amplitude, $L=L_{x}=L_{y}=30$ $\mathrm{km}$ as in Eq. (3.1), $m$ and $n$ are parameters that determine the ellipticity of the storm, $\hat{x}$ and $\hat{y}(=L / 2, L / 4$, respectively) are the initial coordinates of the storm, and $t$ is time $(\times 10 \mathrm{~min})$. The components of the storm motion $C_{x}=0, C_{y}=5(\times 1 \mathrm{~km}$ per $10 \mathrm{~min})$ are defined so that the storm moves outward radially from the radar. Dimensional values of $t, C_{x}$, and $C_{y}$ are irrelevant for these idealizations but have been supplied for completeness. Our objective is to determine how well the analysis preserves storm amplitude. Thus, we choose $C_{y}$ and evaluate Eq. (3.3) at specific times during the interval $0 \leq t \leq 3$ such that maximum of the discretized $g(x, y, t)$ coincides with a data point. To make such collocation more convenient, we redefine the range-gate spacing of our radar to be $\Delta R=0.1 \mathrm{~km}$. Since the storm moves along the radial at $0^{\circ}$ azimuth, sampling of the maximum amplitude is guaranteed, therefore reducing the sampling bias in the error measures.

We limit our discussion to the analyses produced by the Barnes (with $\kappa=2.99 \mathrm{~km}^{2}$, based on a maximum range of $35 \mathrm{~km}$ and thus $\Delta_{\max }=1.22 \mathrm{~km}$ ) and modified Barnes (with $\kappa^{*}=0.5$ ) OA schemes. ${ }^{8}$ Differences in maximum amplitude between the exact, discretized, and objectively analyzed storms are displayed in Fig. 12 over the time interval $0 \leq t \leq 3$ for various values of $m(=n)$ in Eq. (3.3). Examples of the fields themselves are presented in Fig. 13. With the "unmodified" Barnes

\footnotetext{
${ }^{8}$ Owing to its constant radius of influence, our expectation is that the Cressman scheme will behave in a manner similar to that of the traditional Barnes scheme, when applied to this unsteady problem. The design of the experiments does not allow much useful information to be revealed about the behavior of the bilinear interpolation and nearest-neighbor techniques.
} 

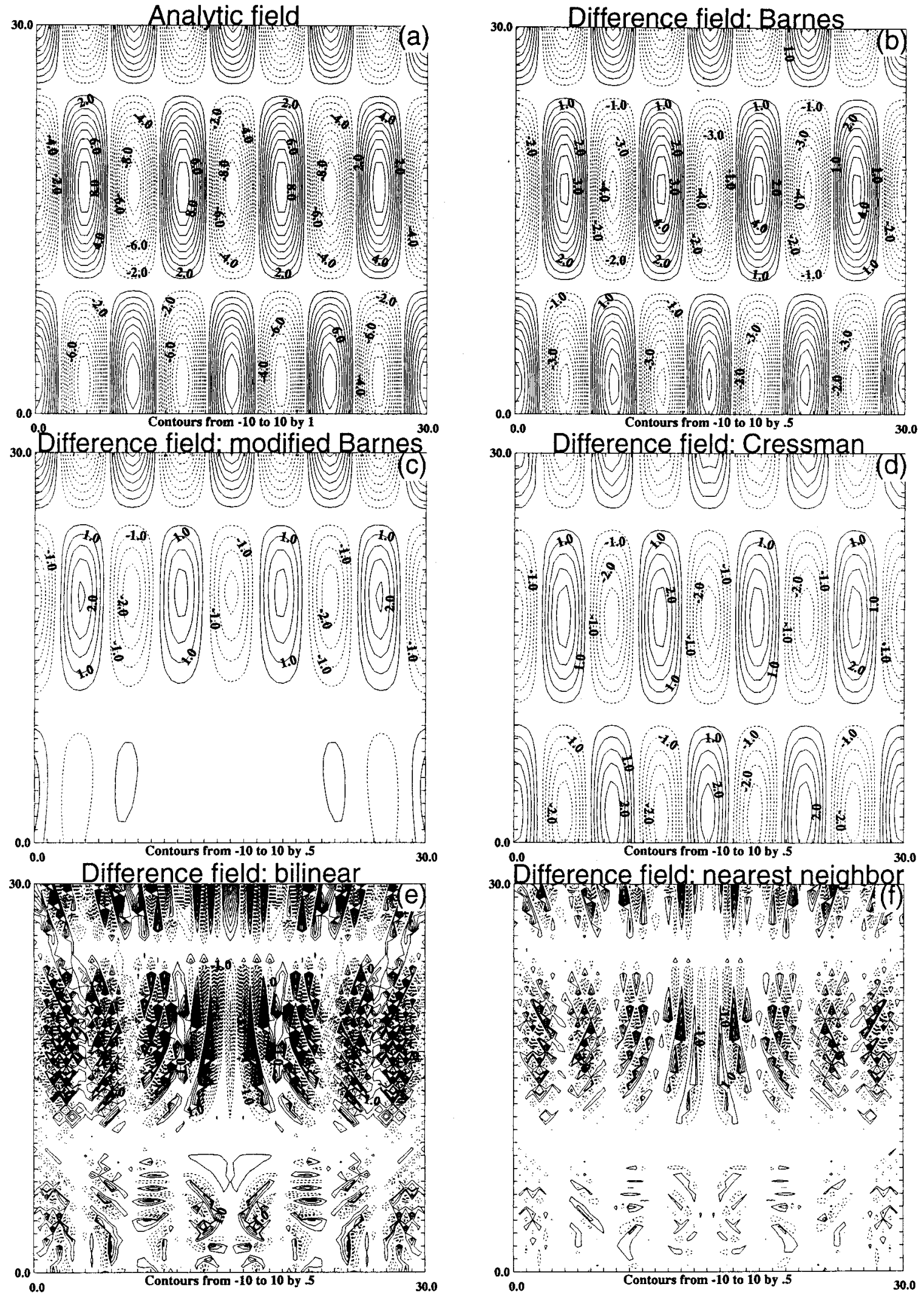

FIG. 9. As in Fig. 7, except for the asymmetric input data case with $(k=4) /(l=1)=4$ in Eq. (3.1). 


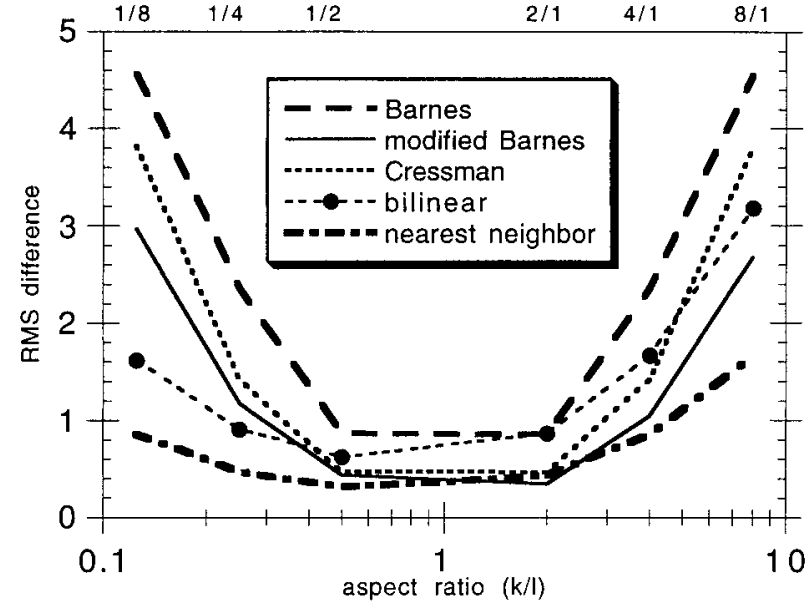

FIG. 10. As in Fig. 6, except with input data defined by Eq. (3.1) for a range of wavenumber index aspect ratio $(=k / l)$. scheme, the amplitude difference or error (as well as the rms difference and MAE) remains relatively constant with time and therefore with variations in azimuthal datapoint spacing (Fig. 12a). This is a predictable result in light of the simple exercise discussed in section $2 \mathrm{~b}$. In contrast, use of the modified Barnes scheme results in amplitude errors that increase nearly monotonically with time (and implicitly, with azimuthal datapoint spacing); the rate of error increase is dependent on parameters $m$ and $n$, the magnitude of which are inversely proportional to the physical scale of the storm (Fig. 12b).

An unsteady, ellipsoidal storm (henceforth, "line") is created when $m=0.5$ and $n=5$ in Eq. (3.3). Figure 14 depicts the objective analyses of the line at two times during the interval $0 \leq t \leq 3$. As in Fig. 13, the damped, yet temporally consistent line due to the traditional Barnes weighting contrasts the more globally accurate, yet temporally varying (in amplitude and now shape) line due to the modified Barnes weighting.

These artifacts of the modified Barnes weight func-
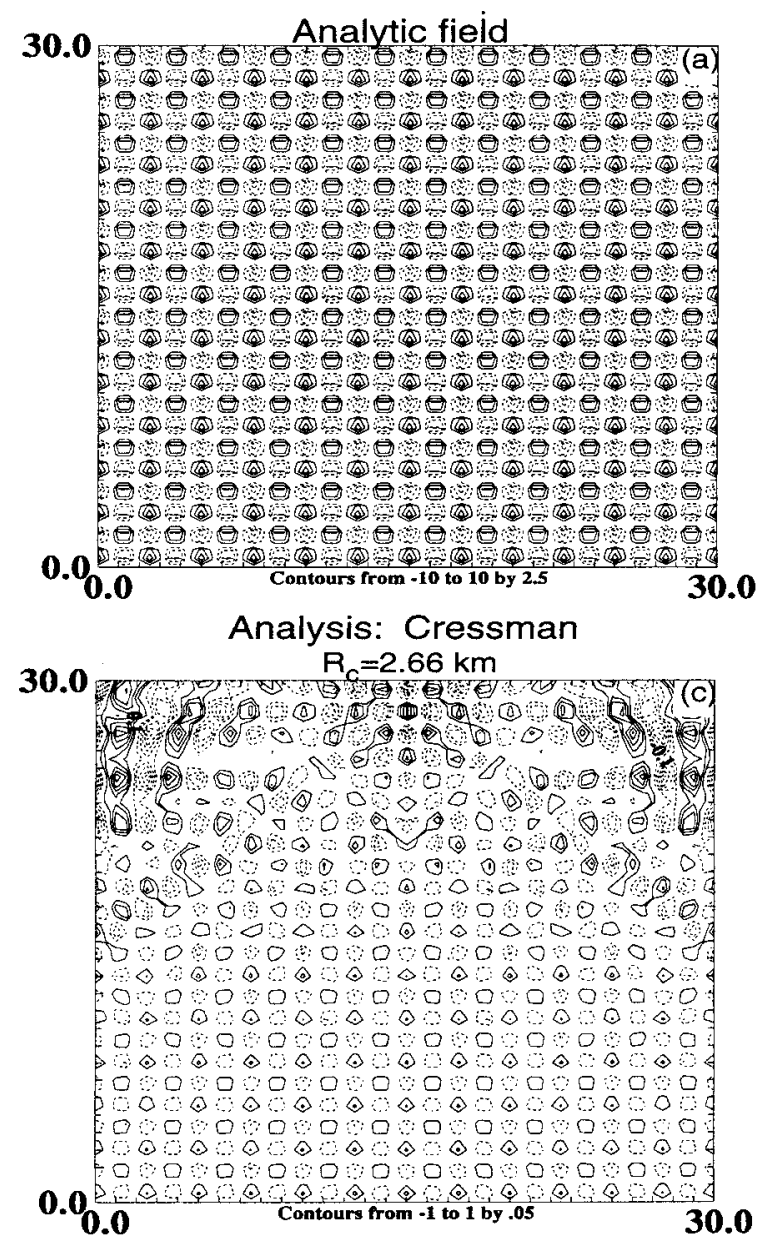

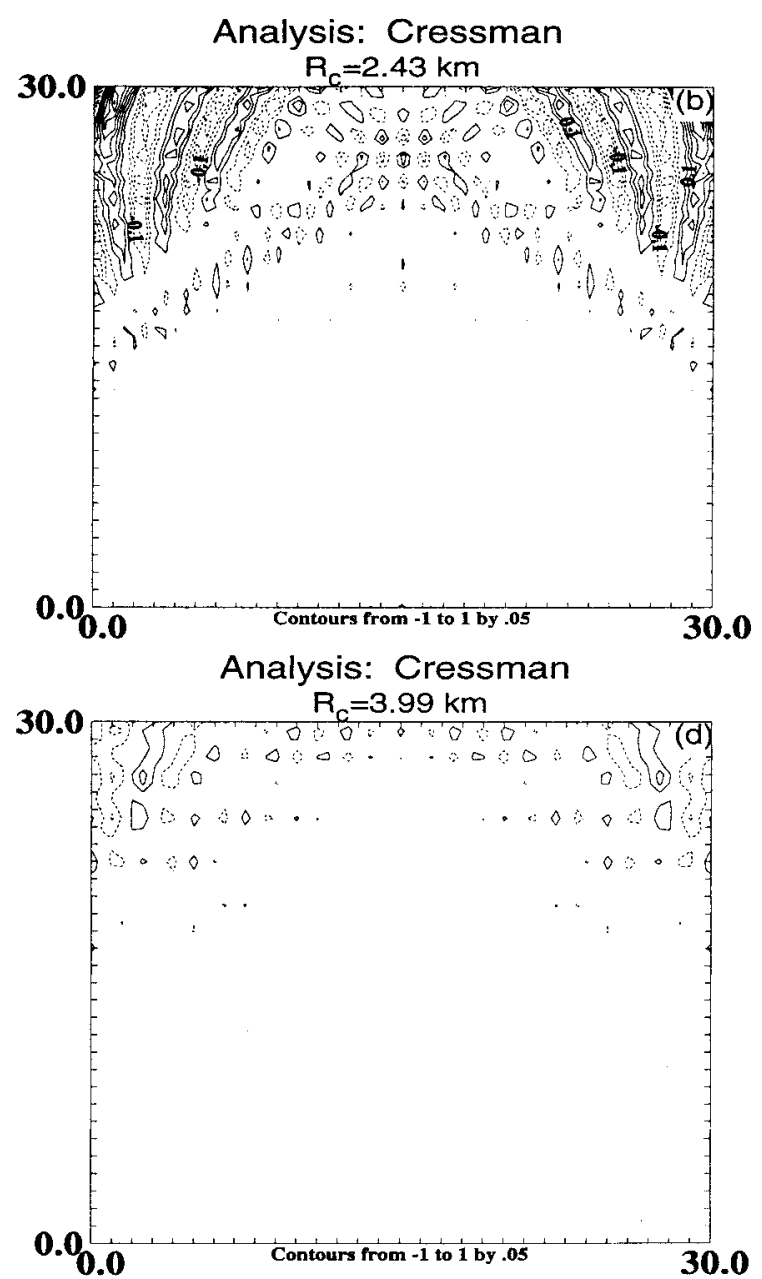

FIG. 11. (a) Discrete evaluation of Eq. (3.1) for the case $k=l=12$. Corresponding objective analyses produced by the Cressman technique with (b) $R_{c}=2.43$, (c) $R_{c}=2.66$, and (d) $R_{c}=3.99 \mathrm{~km}$. 

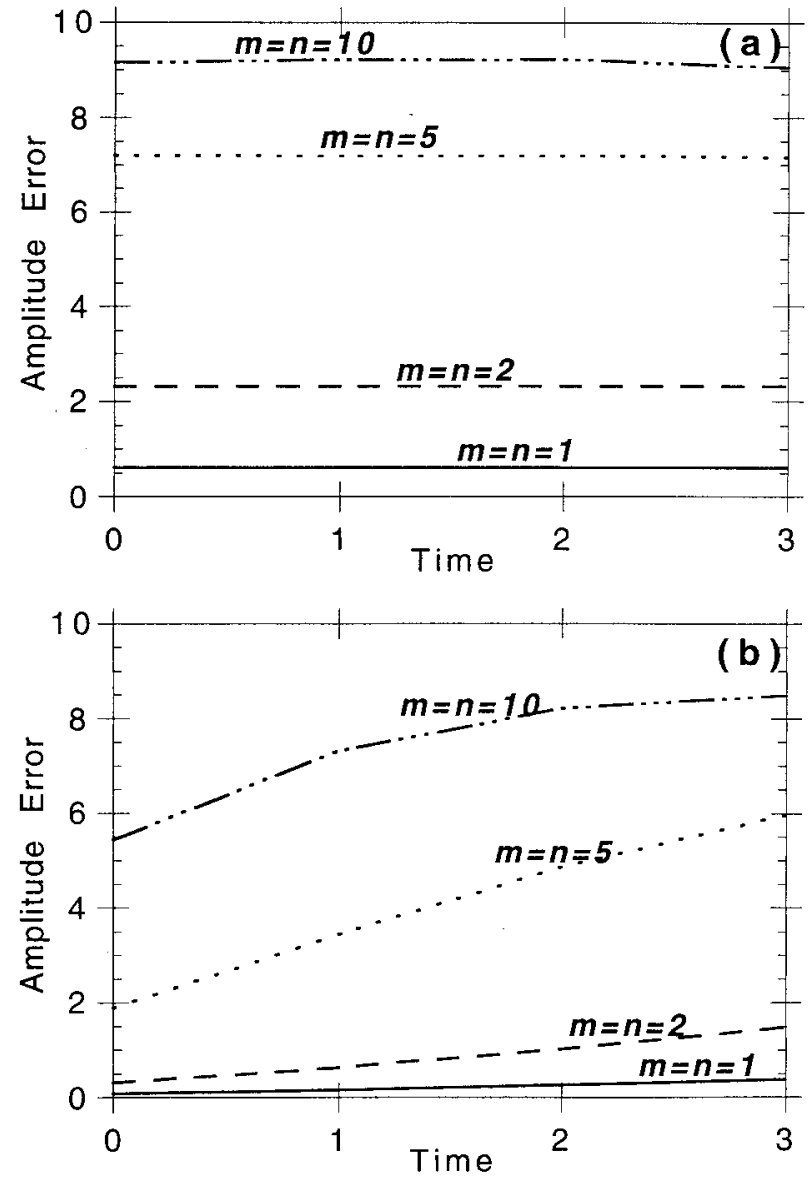

FIG. 12. Error in maximum amplitude in analyses produced by the (a) Barnes and (b) modified Barnes OA techniques, as a function of nondimensional time and input parameter $m(=n)$ in Eq. (3.3).

tion have far-reaching implications. In real-data applications, the spatial variations of this weight function will be convolved with the temporal evolution of the analyzed field so that unambiguous assessment of time tendencies in the analyzed field is not possible. This means that subsequent diagnoses and prognoses based on the analysis will be muddled with this artifact, and has special relevance when gridded radar data are used as initial and/or boundary conditions in mesoscale prediction models. As just shown, a convective storm or line maintaining its intensity while moving away from the radar appears in the analyses to be decaying. This incorrect deduction invalidates the predicted future state of the storm or line. Hence, the penalty for relatively low global error is the potential for misinterpretations of the analysis by its users.

\section{c. Anisotropic weighting}

Datapoint spacing that is vastly different along one coordinate direction (spherical or Cartesian) compared to that along another has been the justification of some analysts to employ DDWA that is dependent on coordinate directions. Armed with a so-called anisotropic weight function, the analyst attempts to retain the greatest "detail" in a given direction, in a manner that is independent from that in another direction.

An anisotropic weight function based on the Barnes scheme in $2 \mathrm{D}$ can be expressed as

$$
w_{q}=\exp \left(\frac{-x^{\prime 2}}{\kappa_{x}}-\frac{y^{\prime 2}}{\kappa_{y}}\right)
$$

where, in the respective $x$ and $y$ directions, $x^{\prime}$ and $y^{\prime}$ are Cartesian distances separating an analysis point from a data point, and $\kappa_{x}$ and $\kappa_{y}$ are the smoothing parameters. When $\kappa_{x}=\kappa_{y}$, Eq. (3.4) reduces to Eq. (2.5). Alternatively, the $2 \mathrm{D}$ weight function can be defined with respect to radial and azimuthal gridpoint-datapoint separation distances $R^{\prime}$ and $\theta^{\prime}$ :

$$
w_{q}=\exp \left(\frac{-R^{\prime 2}}{\kappa_{R}}-\frac{\theta^{\prime 2}}{\kappa_{\theta}}\right),
$$

where $\kappa_{R}$ and $\kappa_{\theta}$ are the smoothing parameters in the radial and azimuthal directions, respectively. Equation
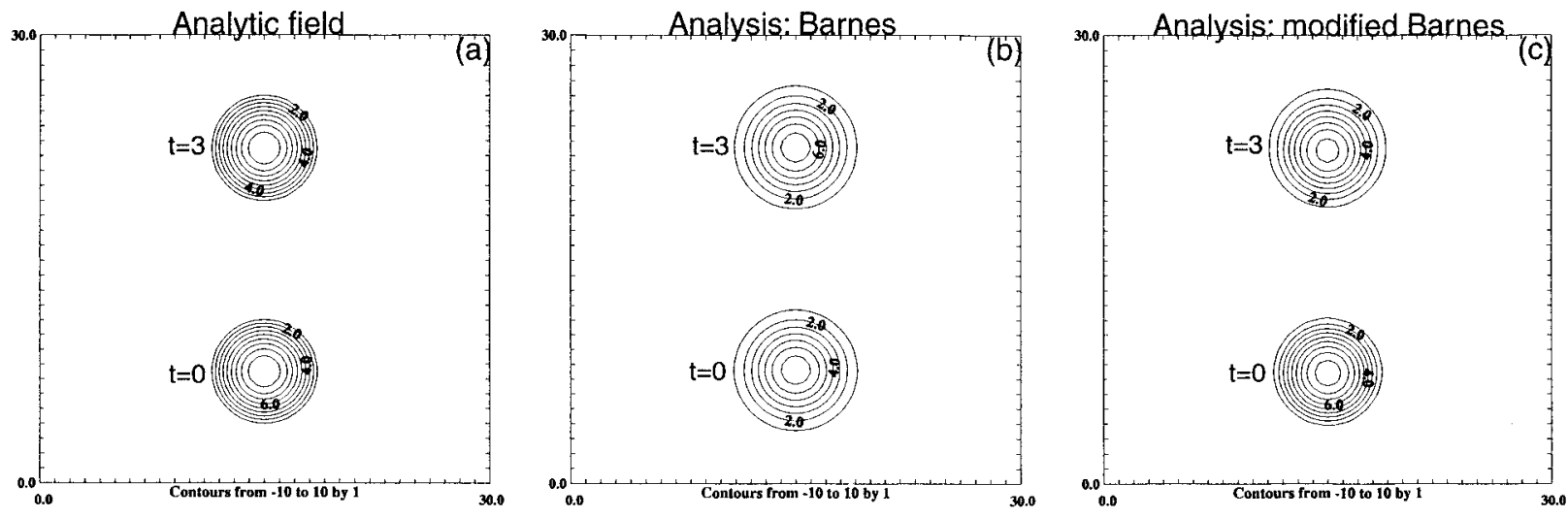

FIG. 13. (a) Discrete evaluation of Eq. (3.3) for the case $m=n=2$, at times $t=0$ and $t=3$, and corresponding objective analyses produced by the (b) Barnes and (c) modified Barnes techniques. 

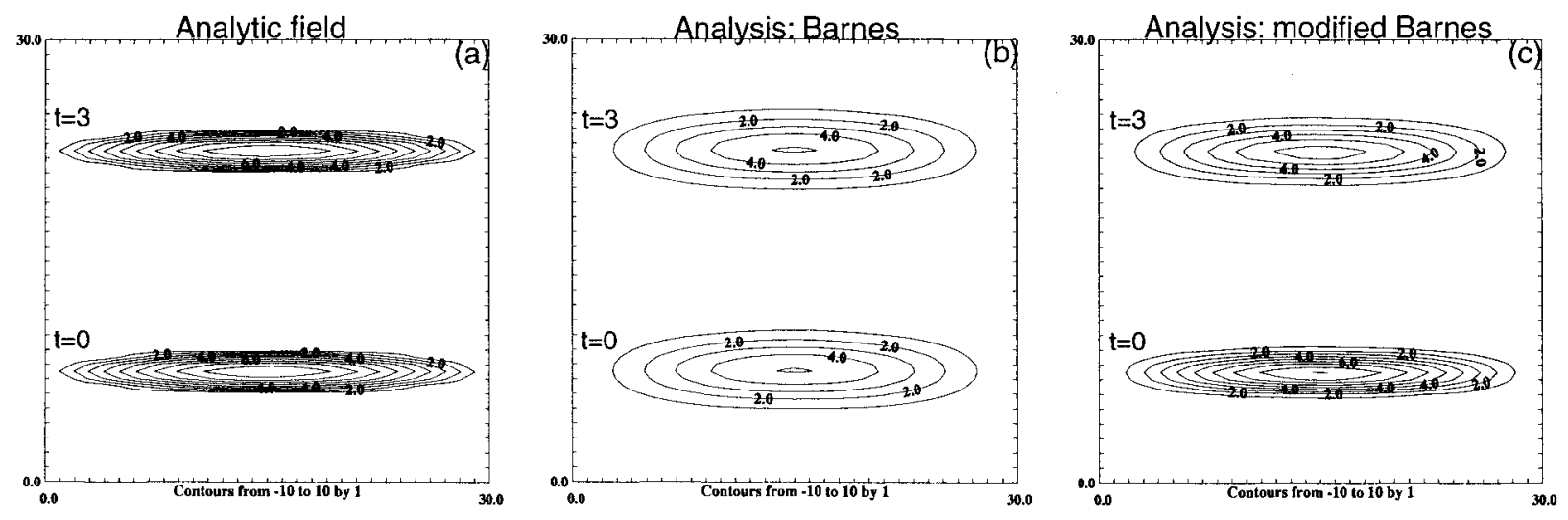

FIG. 14. As in Fig. 13, except for the case $m=0.5, n=5$.

(3.5) follows the 3D formulation of Askelson (1996). Analogous expressions for the Cressman weight function follow from Eq. (2.8).

Our empirical methodology can be used to examine how anisotropic weight functions treat the input data. Consider the data generated by Eq. (3.1) with $k=l=$ 2 , as in experiments presented in section 3a. Objective analyses are obtained using weight function (3.4) with smoothing parameters $\kappa_{x}=3.54 \mathrm{~km}^{2}, \kappa_{y}=0.354 \mathrm{~km}^{2}$ (Fig. 15a), and $\kappa_{x}=0.354 \mathrm{~km}^{2}, \kappa_{y}=3.54 \mathrm{~km}^{2}$ (Fig. $15 \mathrm{~b}$ ); weight function (3.5) is applied with $\kappa_{R}=2.25$ $\times 10^{-2} \mathrm{~km}^{2}$ and $\kappa_{\theta}=4.87 \times 10^{-3}$ (Fig. 15c). The values of $\kappa_{x}$ and $\kappa_{y}$ are computed from Eq. (2.15) using either $\kappa^{*}=0.5$ or $\kappa^{*}=0.05$ with $\Delta=1.33 \mathrm{~km}$; values of $\kappa_{R}$ and $\kappa_{\theta}$ are computed from Eq. (2.15) using $\kappa^{*}=$ 1.0 with $\Delta R=0.075 \mathrm{~km}$ and $\Delta \theta=2^{\circ}$, respectively. Our respective choices for $\kappa_{x}, \kappa_{y}, \kappa_{R}$, and $\kappa_{\theta}$ result in a highly elliptical weight function, purposely exaggerated for the sake of illustration.

Standard measures of error (such as rms or MAE) suggest that these analyses are more accurate than those produced with an isotropic weight function (with $\kappa=3.54 \mathrm{~km}^{2}$ ), owing to reduced smoothing in one direction (see also Fig. 7). Figure 15b results from values of $\kappa_{x}$ and $\kappa_{y}$ that clearly are less suited to the range-varying datapoint spacing than are the values used to produce Fig. 15a. An implicit, range-dependent smoothing property of weight function (3.5) is depicted in the difference field in Fig. 15c. At distant ranges from the radar, for example, the azimuthal changes $\theta^{\prime}$ are smaller over some $x$ distance than are the $\theta^{\prime}$ at near ranges over the same $x$ distance due to the polar geometry of the data. Because the weight function is inversely proportional to $\theta^{\prime}, w$ increases with range.

Figure 15 c illustrates an undesirable property of anisotropic weight functions that is most evident when the input data contain large gradients. We illustrate this with the analytic field given by

$$
h\left(r^{\prime \prime}\right)= \begin{cases}C, & r^{\prime \prime}<7.5 \\ C \frac{10-r^{\prime \prime}}{10-7.5}, & 7.5 \leq r^{\prime \prime} \leq 10 \\ 0, & r^{\prime \prime}>10,\end{cases}
$$

where

$$
r^{\prime \prime}=\left[\left(x-x_{0}\right)^{2}+\left(y-y_{0}\right)^{2}\right]^{1 / 2},
$$

$C=10$ is the constant amplitude, and $x_{0}=y_{0}=L / 2$ define the center of $h$. This function describes a circular region with a large gradient, which might be likened to the surface temperature distribution within a radially symmetric, thunderstorm outflow gust front (henceforth, "front"; see Fig. 16a).

Anisotropic smoothing owing to weight functions (3.4) and (3.5) (with $\kappa_{x}, \kappa_{y}, \kappa_{R}$, and $\kappa_{\theta}$ as in Fig. 15) results in a nonuniform distortion of the circular front (Fig. 16). Other experiments (not shown) indicate that the degree of distortion increases with the anisotropy, which also is exaggerated in these experiments for the sake of illustration. The "character" of the distortion depends on the alignment of the local gradient vector with the major axis of the essentially elliptical weight function (see Figs. 16b,c). Although anisotropic weighting allows for relatively greater accuracy (in terms of standard measures), qualitatively it also may give rise to unintended interpretations about the physics of the analyzed field. In Fig. 16, for example, one might deduce incorrectly that portions of the front have weakened and become more diffuse. More serious misdiagnoses and prognostic errors may arise if anisotropic weighting is used to analyze an unsteady front undergoing a configuration change. We note that there may be some (perhaps 3D) applications, unlike our examples, in which anisotropic weighting might still be desirable. This deserves further study. 


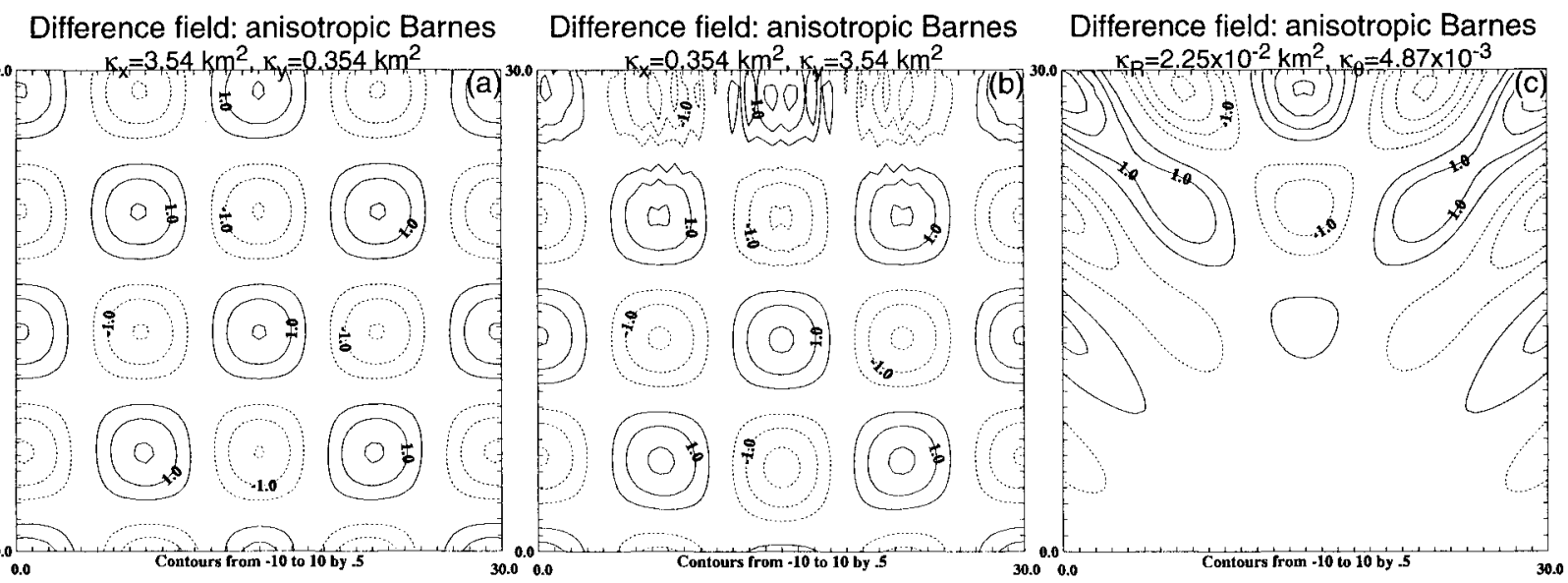

FIG. 15. As in Fig. 7, except for difference fields between the discretized, exact function and analyses produced using the (a) anisotropic Barnes weight function (3.4) with $\kappa_{x}=3.54 \mathrm{~km}^{2}$ and $\kappa_{y}=0.354 \mathrm{~km}^{2}$, (b) anisotropic Barnes weight function (3.4) with $\kappa_{x}=0.354 \mathrm{~km}{ }^{2}$ and $\kappa_{y}=3.54 \mathrm{~km}^{2}$, and (c) anisotropic Barnes weight function (3.5) with $\kappa_{R}=2.25 \times 10^{-2} \mathrm{~km}^{2}, \kappa_{\theta}=4.87 \times 10^{-3}$.
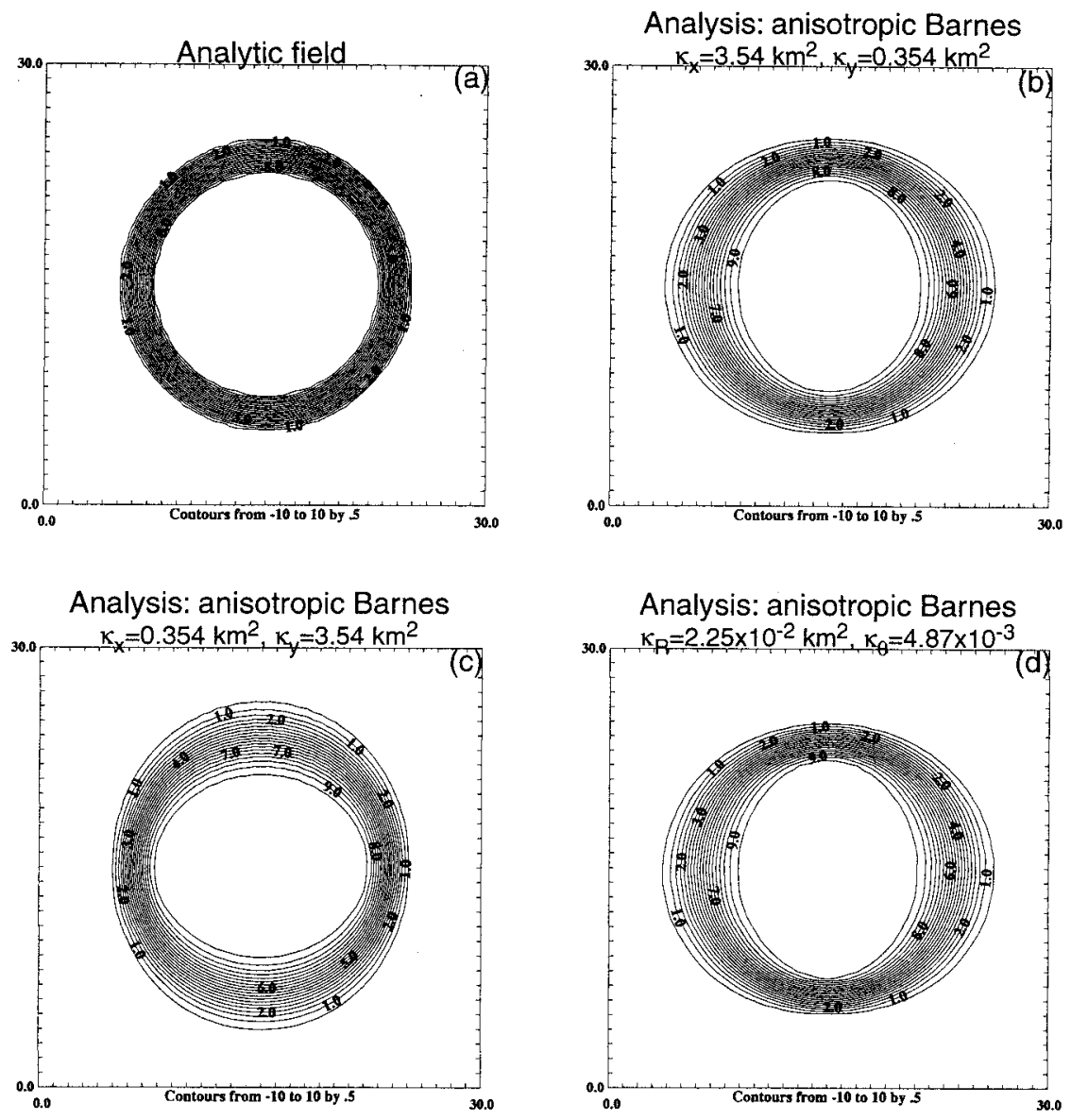

FIG. 16. (a) Discrete evaluation of Eq. (3.6), and corresponding objective analyses produced by anisotropic Barnes weight functions with smoothing parameter values of (b) $\kappa_{x}=3.54 \mathrm{~km}^{2}$, $\kappa_{y}=0.354 \mathrm{~km}^{2}$ in Eq. (3.4), (c) $\kappa_{x}=0.354 \mathrm{~km}^{2}, \kappa_{y}=3.54 \mathrm{~km}^{2}$ in Eq. (3.4), and (d) $\kappa_{R}=2.25$ $\times 10^{-2} \mathrm{~km}^{2}, \kappa_{\theta}=4.87 \times 10^{-3}$ in Eq. (3.5). 


\section{Summary and discussion}

Results from the preceding examination of radar data objective analysis (RDOA) techniques can be summarized as follows.

1) Isotropic, distant-dependent weighted-averaging (DDWA) schemes with Cressman or Barnes weight functions that have constant "shape" parameters (i.e., Barnes smoothing parameter or Cressman radius of influence) filter or damp amplitudes of dimensional wavelengths irrespective of the datapoint spacing. This implies loss of detail in the analysis where a wave or feature is well sampled, but also a consistency in how it is treated throughout the analysis domain.

2) Isotropic, DDWA schemes with parameters that vary with the local datapoint spacing treat nondimensional wavelengths the same throughout the analysis domain, but filter/smooth amplitudes of locally dimensionalized wavelengths based on the local datapoint spacing. Hence, waves that are well resolved within the varying datapoint distribution are well-retained in the analysis. One consequence of such a spatially varying weight function is that the associated spatial variation in amplitude error may be misinterpreted as some physical change in the analyzed field. In particular, analyses of unsteady meteorological fields suffer from a convolution of the evolution of the input field with spatial variations of the weight function. Subsequent diagnoses and prognoses based on these analyses will be muddled with this artifact.

3) The Cressman weight function generates negative values of response, or "sidelobes," in the spectral domain. According to theory based on continuous data distributions, these lead to an analysis whose phase in the short wavelength part of the spectral domain is shifted with respect to that of the input field. The sidelobes occur at progressively larger wavelengths as the Cressman influence radius increases. As a consequence, wavelengths that are filtered with a relatively narrow weight function can be readmitted into the analysis when a broader weight function is applied.

4) DDWA schemes with anisotropic weights can distort the shapes of the input field. The associated analyses are sensitive to the orientation of the input field with respect to the orientation of the weight function itself. This artifact also complicates subsequent diagnoses and prognoses.

5) Bilinear and "nearest-neighbor" interpolation schemes generate wavelengths not present in the initial data. Analyses via these schemes [as well as the DDWA scheme in (2)] are inherently sensitive to characteristics of the input field (e.g., orientation of the phenomenon) with respect to the datapoint distribution.

In terms of preservation of the phase and amplitude of the input data, predictability of the resultant smoothing and filtering, and relative insensitivity to input data unsteadiness or spatial characteristic, the isotropic Barnes weight function with constant smoothing parameter appears to be the most desirable of the schemes considered. Recalling that there is no single Nyquist wavelength in radar data, we recommend that the smoothing parameter be defined with respect to the maximum datapoint spacing affecting the analysis domain. This "conservative" viewpoint avoids the potential for undersmoothing in regions of the most coarse data resolution. The choice of nondimensional smoothing parameter $\kappa^{*}$, hence, the degree of smoothing near the limits of resolution is, as always, related to the philosophy and intentions of the analyst.

We note that OA is not a data panacea. This is expressed by Mohr and Vaughn (1979) who, in a discussion of artifacts due to inadequate resolution, state that "More elaborate interpolation schemes were examined and as the degree of sophistication increased the artifacts became smoother, but they never disappeared." Indeed, there can be no substitute for data resolution.

Several issues pertinent to objective analysis methodology were not treated here. The choice of analysis domain size for a given radar data domain size, and the ramifications this has on the analysis at the domain boundary is one such issue (see Achtemeier 1986; Pauley 1990). Another is the choice of gridpoint spacing. These merit further study.

A closing remark is appropriate. Our conclusions above should not be interpreted to mean that the only "correct" RDOA scheme is the one that employs an isotropic Barnes-type weight function with a constant smoothing parameter. The choices of the 1) OA scheme and 2) its coefficients or parameters are problem-specific. It is the responsibility of the analyst to understand the interaction between the data and the various OA schemes and make an informed decision on which technique is most appropriate for the intended purpose of the analysis.

Acknowledgments. The authors have benefited from discussions with Mark Askelson and Erik Rasmussen. Comments on a draft of this manuscript were provided by Phillip Spencer and Rodger Brown. This work was initiated while the first author was a National Research Council-NOAA Postdoctoral Research Associate, and completed while he was a Visiting Scientist with the Mesoscale and Microscale Meteorology Division of the National Center for Atmospheric Research. The National Center for Atmospheric Research is sponsored by the National Science Foundation.

\section{REFERENCES}

Achtemeier, G. L., 1986: The impact of data boundaries upon a successive corrections objective analysis of limited-area datasets. Mon. Wea. Rev., 114, 40-49. 
Askelson, M. A., 1996: A study of the kinematic and microphysical evolution of a supercell from first echo using polarization diversity radar. M.S. final report, University of Oklahoma, 94 pp. [Available from School of Meteorology, University of Oklahoma, 100 E. Boyd, Norman, OK 73019.]

Barnes, S. L., 1964: A technique for maximizing details in numerical weather map analysis. J. Appl. Meteor., 3, 396-409.

Bluestein, H. B., S. G. Gaddy, D. C. Dowell, A. L. Pazmany, J. C. Galloway, R. E. McIntosh, and H. Stein, 1997: Doppler radar observations of substorm-scale vortices in a supercell. Mon. Wea. Rev., 125, 1046-1059.

Brown, R. A., and V. T. Wood, 1998: Technique for improving WSR$88 \mathrm{D}$ detection of tornadoes. Preprints, 19th Conf. on Severe Local Storms, Minneapolis, MN, Amer. Meteor. Soc., 721-724.

Carbone, R. E., M. J. Carpenter, and C. D. Burghart, 1985: Doppler radar sampling limitations in convective storms. J. Atmos. Oceanic Technol., 2, 357-361.

Cressman, G. P., 1959: An operational objective analysis system. Mon. Wea. Rev., 87, 367-374.

Daley, R., 1991: Atmospheric Data Analysis. Cambridge University Press, $457 \mathrm{pp}$.

Errico, R. M., 1985: Spectra computed from a limited area grid. Mon. Wea. Rev., 113, 1554-1562.

Heymsfield, G. M., 1976: Statistical objective analysis of dual-Doppler radar data from a tornadic storm. J. Appl. Meteor., 15, 5968.

Hildebrand, P. H., and Coauthors, 1996: The ELDORA/ASTRAIA airborne Doppler weather radar: High resolution observations from TOGA COARE. Bull. Amer. Meteor. Soc., 76, 213-232.

Jorgensen, D. P., T. Matejka, and J. D. DuGranrut, 1996: Multi-beam techniques for deriving wind fields from airborne Doppler radars. J. Meteor. and Atmos. Phys., 59, 83-104.

Koch, S. E., M. DesJardins, and P. J. Kocin, 1983: An interactive Barnes objective analysis scheme for use with satellite and conventional data. J. Climate Appl. Meteor., 22, 1487-1503.

Mohr, C. G., and R. L. Vaughan, 1979: An economical procedure for Cartesian interpolation and display of reflectivity factor in threedimensional space. J. Appl. Meteor., 18, 661-670.

Nelson, S. P., 1980: A study of hail production in a supercell storm using a Doppler-derived wind field and a numerical hail growth model. NOAA Tech. Memo. ERL NSSL-89, National Severe Storms Laboratory, 90 pp. [NTIS PB81-17822Q.]

Pauley, P. M., 1990: On the evaluation of boundary errors in the Barnes objective analysis scheme. Mon. Wea. Rev., 118, 12031210.

— , and X. Wu, 1990: The theoretical, discrete, and actual response of the Barnes objective analysis scheme for one- and two-dimensional fields. Mon. Wea. Rev., 118, 1145-1210.

Press, W. H., B. P. Flannery, S. A. Teukolsky, and W. T. Vetterling, 1986: Numerical Recipes. Cambridge University Press, 818 pp.

Rasmussen, E. N., J. M. Straka, R. P. Davies-Jones, C. A. Doswell, F. H. Carr, M. D. Eilts, and D. R. MacGorman, 1994: Verification of the Origins of Rotation in Tornadoes Experiment: VORTEX. Bull. Amer. Meteor. Soc., 75, 995-1006.

Scialom, G., and Y. Lemaître, 1990: A new analysis for the retrieval of three-dimensional mesoscale wind fields from multiple Doppler radar. J. Atmos. Oceanic Technol., 7, 640-665.

Shapiro, A., L. Zhao, S. Weygandt, K Brewster, S. Lazarus, and K. K. Droegemeier, 1996: Initial forecast fields created from singleDoppler wind retrieval, thermodynamic retrieval and ADAS. Preprints, 11th Conf. on Numerical Weather Prediction, Norfolk, VA, Amer. Meteor. Soc., 119-121.

Testud, J., and M. Chong, 1983: Three-dimensional wind field analysis from dual-Doppler radar data. Part I: Filtering, interpolating, and differentiating the raw data. J. Climate Appl. Meteor., 22, 1204-1215

Trapp, R. J., 1999: Observations of nontornadic low-level mesocyclones and attendant tornadogenesis failure during VORTEX. Mon. Wea. Rev., 127, 1693-1705.

Wakimoto, R. M., and C. Liu, 1998: The Garden City, Kansas, storm during VORTEX 95. Part II: The wall cloud and tornado. Mon. Wea. Rev., 126, 393-408.

- W.-C. Lee, H. B. Bluestein, C.-H. Liu, and P. H. Hildebrand, 1996: ELDORA observations during VORTEX 95. Bull. Amer. Meteor. Soc., 77, 1465-1481.

Wurman, J., J. Straka, E. Rasmussen, M. Randall, and A. Zahrai, 1997: Design and deployment of a portable, pencil-beam, pulsed, 3-cm Doppler radar. J. Atmos. Oceanic Technol., 6, 1502-1512. 